\title{
Microstructure and Plasticity Evolution During Lüders Deformation in an Fe-5Mn-0.1C Medium-Mn Steel
}

\author{
Motomichi KOYAMA, ${ }^{1,2,3) *}$ Takayuki YAMASHITA, ${ }^{4}$ Satoshi MOROOKA, ${ }^{5)}$ Takahiro SAWAGUCHI, ${ }^{6)}$ \\ Zhipeng YANG, ${ }^{1,2)}$ Tomohiko HOJO, ${ }^{1,2)}$ Takuro KAWASAKI ${ }^{\text {() }}$ and Stefanus HARJO ${ }^{\text {() }}$ \\ 1) Institute for Materials Research, Tohoku University, 2-1-1 Katahira, Aoba-ku, Sendai, 980-8577 Japan. \\ 2) Graduate School of Engineering, Tohoku University, 6-6 Aramaki Aza Aoba, Aoba-ku, Sendai, Miyagi, 980-8579 Japan. \\ 3) Elements Strategy Initiative for Structural Materials (ESISM), Kyoto University, Yoshida-honmachi, Sakyo-ku, Kyoto, 606- \\ 8501 Japan. \\ 4) Joining and Welding Research Institute, Osaka University, 11-1 Mihogaoka, Ibaraki, Osaka, 567-0047 Japan. \\ 5) Materials Science Research Center, Japan Atomic Energy Agency, 2-4 Shirataka, Tokai-mura, Naka-gun, Ibaraki, 319-1195 \\ Japan. \\ 6) National Institute for Materials Science, 1-2-1 Sengen, Tsukuba, Ibaraki, 305-0047 Japan. \\ 7) J-PARC Center, Japan Atomic Energy Agency, 2-4 Shirakata, Tokai, Naka, Ibaraki, 319-1195 Japan.
}

(Received on October 19, 2021; accepted on November 19, 2021; J-STAGE Advance published date: February 3, 2022)

\begin{abstract}
The local plasticity and associated microstructure evolution in Fe-5Mn-0.1C medium-Mn steel (wt.\%) were investigated in this study. Specifically, the micro-deformation mechanism during Lüders banding was characterized based on multi-scale electron backscatter diffraction measurements and electron channeling contrast imaging. Similar to other medium-Mn steels, the Fe-5Mn-0.1C steel showed discontinuous macroscopic deformation, preferential plastic deformation in austenite, and deformation-induced martensitic transformation during Lüders deformation. Hexagonal close-packed martensite was also observed as an intermediate phase. Furthermore, an in-situ neutron diffraction experiment revealed that the pre-existing body-centered cubic phase, which was mainly ferrite, was a minor deformation path, although ferrite was the major constituent phase.
\end{abstract}

KEY WORDS: medium-Mn steel; Lüders deformation; local plasticity; martensitic transformation; neutron diffraction.

\section{Introduction}

Medium-Mn steels, which mainly consist of ferrite and metastable austenite, show excellent mechanical performance mainly owing to the transformation-induced plasticity (TRIP) effect. Medium-Mn TRIP steels have been found to demonstrate heterogeneous plastic deformation. ${ }^{1)}$ In particular, cold-rolled and intercritically annealed medium-Mn steels show distinct evolution of Lüders ${ }^{2,3)}$ and Portevin-Le Chatelier ${ }^{4,5)}$ bands after yielding. The behavior of the macroscopically heterogeneous deformation has been noted in order to elucidate the characteristic stress-strain response in the early plasticity stage.

Lüders deformation occurs in carbon-containing steels, ${ }^{6,7)}$ fine-grained metallic materials, ${ }^{8-10)}$ and highly work-hardenable materials. ${ }^{11)}$ The cold-rolled intercritically annealed medium-Mn steels also contain carbon, have a fine-grained

\footnotetext{
* Corresponding author: E-mail: koyama@imr.tohoku.ac.jp
}

microstructure, and show a high work-hardening capability. ${ }^{1,12)}$ Furthermore, the Lüders deformation of medium-Mn steels is characterized by deformation-induced martensitic transformation, ${ }^{13,14)}$ microscopic strain partitioning, ${ }^{15)}$ and macroscopic plasticity discontinuity. ${ }^{16)}$ These three factors strongly correlate with each other. In other words, the deformation-induced martensitic transformation is the primary plasticity mechanism in retained austenite, which enhances the degree of microscopic strain partitioning. The microscopic strain partitioning results in a macroscopically localized deformation path, which causes discontinuous Lüders band propagation (macroscopic plasticity discontinuity). Lüders banding causes surface relief, which further aids the plasticity localization associated with deformation-induced martensitic transformation. Therefore, characterizing and correlating these three factors are essential to understanding plasticity heterogeneity in specific medium-Mn steels.

In this context, we focus on Fe-5Mn-0.1C steel (mass\%), which is a representative medium-Mn steel. ${ }^{1,17-19)} \mathrm{Fe}-5 \mathrm{Mn}-$ 
0.1C steel, with some minor elements, has been considered a model for next-generation high-strength ductile steels. ${ }^{20,21)}$ In particular, the relatively low $\mathrm{Mn}$ and $\mathrm{C}$ contents in the chemical composition range of medium-Mn steels are practically important in terms of cost performance. Using coldrolled and intercritically annealed Fe-5Mn- $0.1 \mathrm{C}$ steel, we attempted to reveal the multi-scale deformation heterogeneity, preferential deformation site, and austenite plasticity mechanism during Lüders deformation. By comparison with previous results obtained using other medium-Mn steels, the general plasticity phenomena in medium-Mn steels were also clarified.

\section{Experimental Procedure}

Fe-4.91Mn-0.092C steel (mass $\%$ ) was prepared by melting. The ingot was hot-rolled at $1200^{\circ} \mathrm{C}$ (finishing temperature was above $850^{\circ} \mathrm{C}$ ) to reduce the thickness by $8 \mathrm{~mm}$, and subsequently air-cooled. The hot-rolled bar was then cold-rolled from the thickness of $8 \mathrm{~mm}$ to that of $2 \mathrm{~mm}$. The plates were then annealed at $650^{\circ} \mathrm{C}$ for $30 \mathrm{~min}$; Fig. 1 shows the as-annealed microstructure. The initial microstructure consisted of a face-centered cubic (FCC) phase, consisted of austenite, and a body-centered cubic (BCC) phase, which consisted of mainly ferrite because the image quality was high and the lath structure was not observed in most of the BCC regions. The annealed plates were cut by spark machining and mechanically ground to produce the specimens, as shown in Fig. 2(a).

Tensile tests were carried out at $21^{\circ} \mathrm{C}$ with initial strain rates of $10^{-2}, 10^{-3}, 10^{-4}$, and $10^{-5} \mathrm{~s}^{-1}$. A tensile test conducted at an initial strain rate of $10^{-4} \mathrm{~s}^{-1}$ was interrupted at a strain of $5 \%$. Strains were measured using a video extensometer. The specimen deformed by $5 \%$ was mechanically polished using $\mathrm{SiC}$ grinding paper, diamond suspensions with particle sizes of $9 \mu \mathrm{m}$ and $3 \mu \mathrm{m}$, and colloidal silica with a particle size of $60 \mathrm{~nm}$ before the tensile test, and further mechanical polishing was conducted after the 5\% deformation using the $9 \mu \mathrm{m}$ and $3 \mu \mathrm{m}$ diamond suspensions, and the $60 \mathrm{~nm}$ colloidal silica. The polished surface was observed by electron channeling contrast imaging (ECCI) and electron backscatter diffraction (EBSD) analysis carried out at acceleration voltages of 30 and $20 \mathrm{kV}$, respectively.

In-situ neutron diffraction measurements during a tensile test was conducted at $25^{\circ} \mathrm{C}$ with an initial strain rate of $2.0 \times$

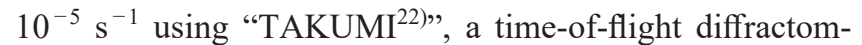
eter dedicated to the investigation of engineering materials at the MLF of J-PARC. The tensile loading direction was horizontally aligned at $45^{\circ}$ to the incident neutron beam. The neutron diffraction data for the scattering vector parallel to the loading direction were collected at the axial detector, while the data for the scattering vector perpendicular to the loading axis were collected at the transverse detector. An incident beam slit of dimensions $5 \mathrm{~mm}$ (width) $\times 5 \mathrm{~mm}$ (height) was employed, and a pair of radial collimators viewing $5 \mathrm{~mm}$ width was adopted. The proton beam power for neutron diffraction measurements was $600 \mathrm{~kW}$. The specimen geometry for the in-situ neutron diffraction experiment is shown in Fig. 2(b). The neutron diffraction data were sliced every $300 \mathrm{~s}$, and neutron diffraction measurements were performed until the ultimate tensile strength was

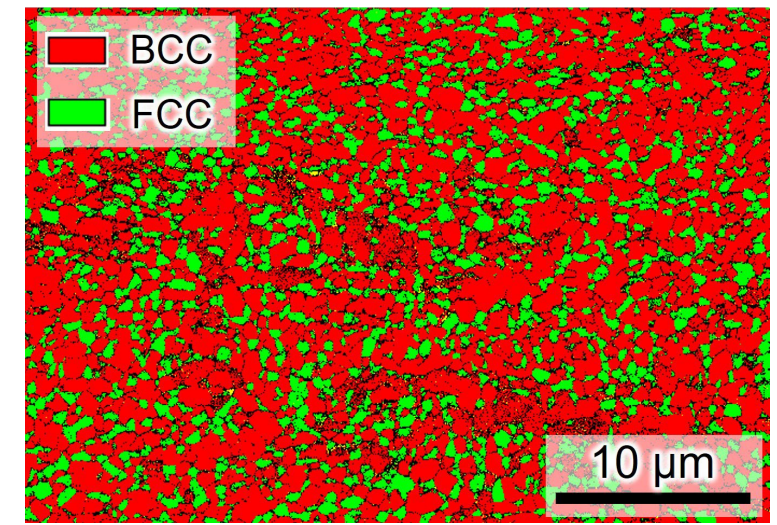

Fig. 1. Initial microstructure consisting of ferrite and austenite The austenite area fraction is $21 \%$. The beam step size is $50 \mathrm{~nm}$. The regions where confidence index is below 0.1 are show in black. (Online version in color.)

(a)

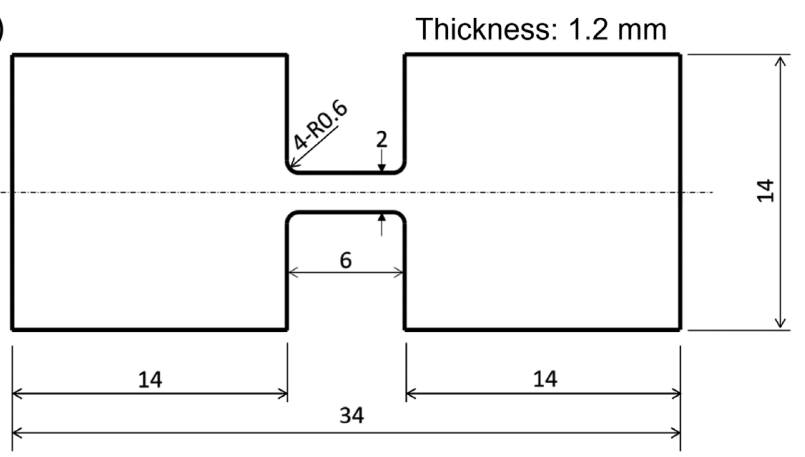

(b)

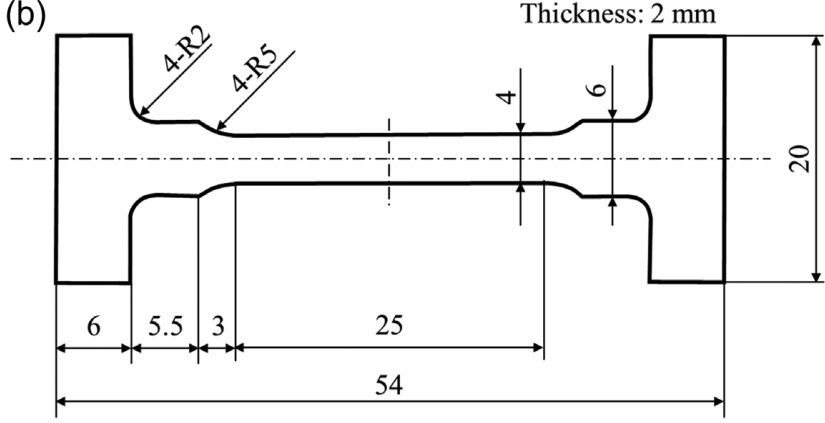

Fig. 2. Specimen geometries used for (a) the normal tensile tests and (b) the in-situ neutron diffraction experiment (unit: $\mathrm{mm})$.

reached. The full width at half maximum (FWHM) values of several hkl planes of ferrite and austenite in the loading direction were obtained using a single peak-fitting method available in the Z-Rietveld software. ${ }^{23)}$ Peak separation between ferrite and deformation-induced martensite was then conducted by assuming that the c/a ratio of martensite was constant during the entire deformation process and could be simplified by a single-broad BCC peak.

\section{Results and Discussion}

\subsection{Stress-strain Response}

Figure 3 shows the engineering stress-strain curves at various strain rates. Although the flow stress did not change significantly with the strain rate, a distinct serrated flow appeared particularly at low strain rates, arising reportedly from dynamic strain aging. ${ }^{4)}$ In addition, Lüders deforma- 
tion occurred irrespective of the strain rate, i.e. Lüders elongation did not change when the strain rate was varied. In this study, we focus on the Lüders band morphology and the associated microstructure evolution.

\subsection{Morphologies of Lüders Front and Associated Deformation Bands}

The ECC image taken around the Lüders front at 5\% engineering strain, where the Lüders deformation was about to end (Fig. 4(a)), showed that the deformation was heterogeneous and discontinuous. Therefore, it was difficult to define the exact position of the Lüders front. Alternatively, the dense deformation band region was considered

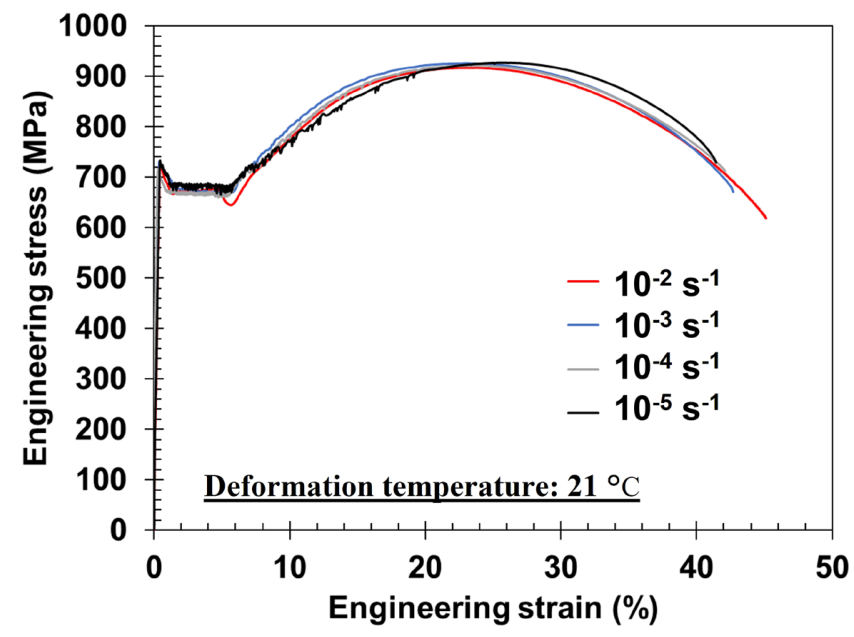

Fig. 3. Engineering stress-strain curves obtained at different strain rates. (Online version in color.) the Lüders front in this study. Numerous deformation bands were also observed in front of the Lüders front. The deformation bands are coarsely distributed, as indicated by the black arrows. In addition, fine deformation bands were formed between the coarse deformation bands, as indicated by the yellow arrows. The magnified image of the Lüders front (Fig. 4(b)) shows that this region consists of dense, fine deformation bands. Because the bright contrast appearing in the ECC image was not observed in the secondary electron images (Fig. 4(c)), the change in contrast corre-
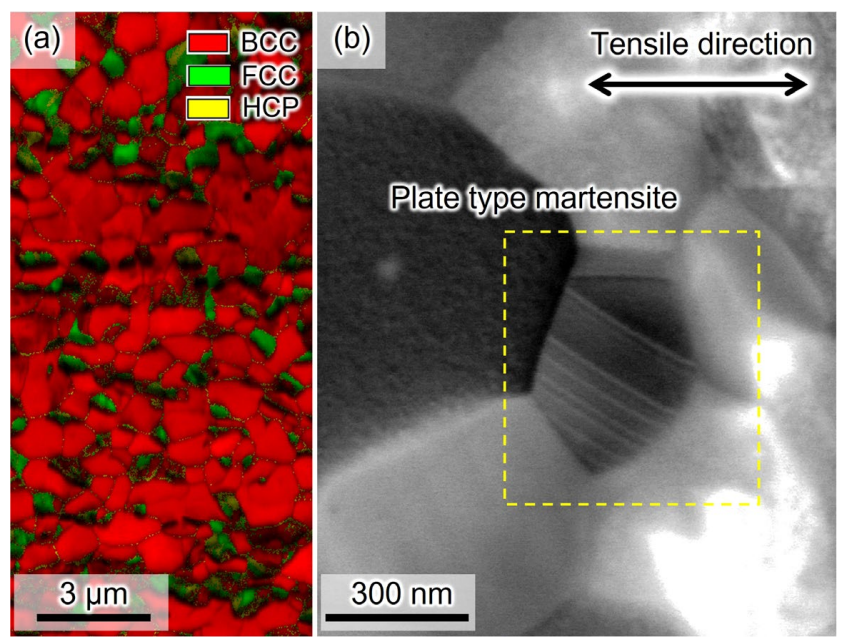

Fig. 5. (a) EBSD phase map of the region where the Lüders band fully propagated. The fraction of austenite in this region is $9 \%$. The beam step size is $30 \mathrm{~nm}$. The regions where confidence index is below 0.1 are shown in black. (b) ECC image of the Lüders front region. (Online version in color.)

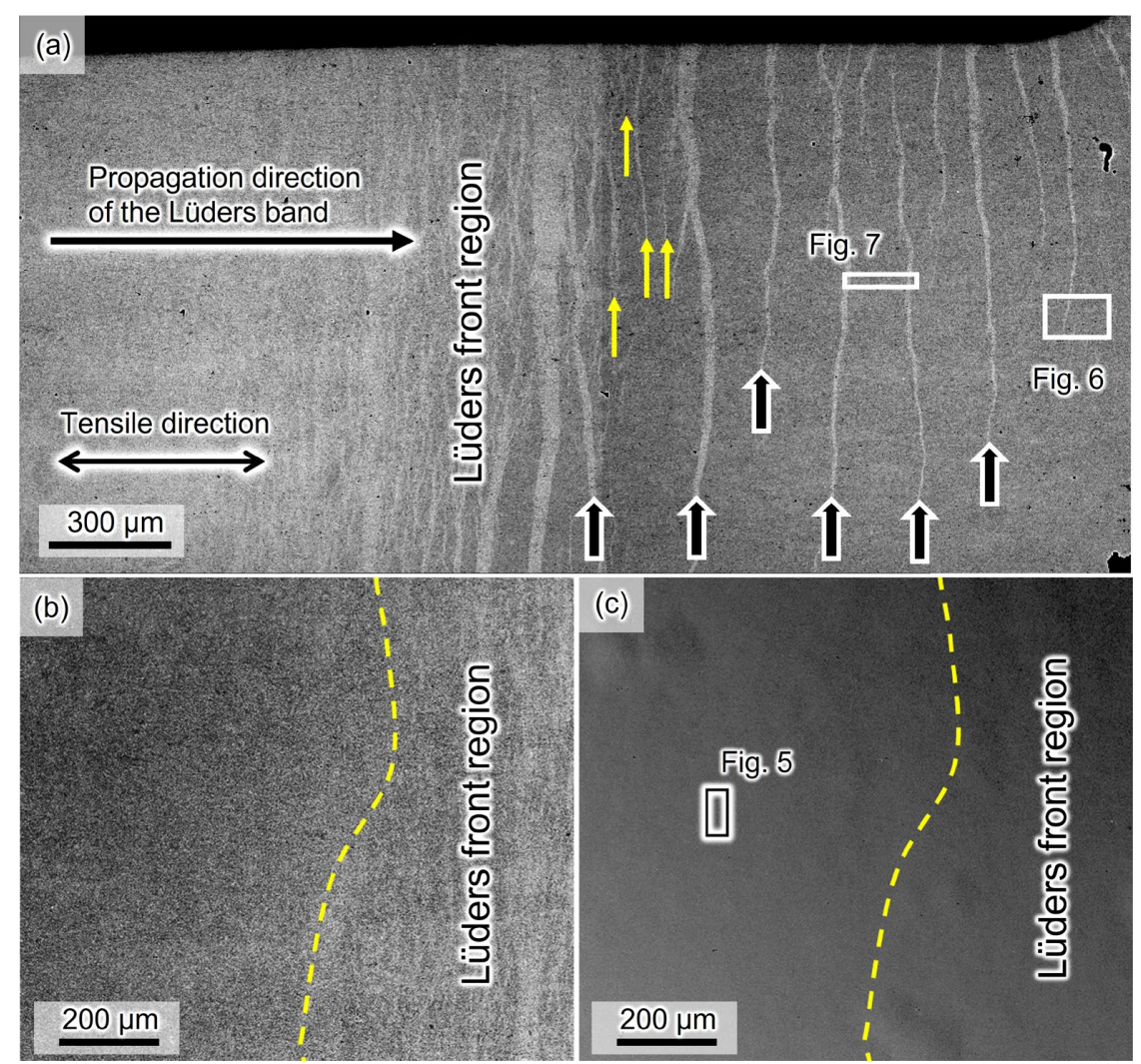

Fig. 4. ECC images showing (a) an overview and (b) a magnified image of the Lüders front. (c) Secondary electron image at the location identical to (b). The black and yellow arrows in (a) indicate coarse and fine deformation bands, respectively. (Online version in color.) 
sponding to the deformation band evolution was attributed to the internal microstructural changes.

A phase map of the region where the Lüders band fully propagated (Fig. 5(a)) showed that the austenite fraction in this region was $9 \%$. Since the initial fraction of austenite was $21 \%$, the microstructural change associated with the deformation band evolution was martensitic transformation from FCC to BCC or body-centered tetragonal (BCT) phases, which is the same as the other medium-Mn steels. ${ }^{13,14)}$ Hereafter, BCC or BCT martensite is referred to as BCC martensite. Hexagonal close-packed (HCP) martensite was also detected by EBSD, as shown in the phase map. Furthermore, the Lüders front region, which underwent early plasticity evolution during Lüders deformation, showed plate-type martensite (Fig. 5(b)), which has been reported to be HCP martensite in medium-Mn steels. ${ }^{2)}$ These results indicated that a portion of the $\mathrm{BCC}$ martensite was formed in the $\mathrm{FCC} \rightarrow \mathrm{HCP} \rightarrow \mathrm{BCC}$ sequence.

\subsection{Martensitic Transformation and Micro-plasticity Evolution during Lüders Deformation}

Next, we focused on the microstructure evolution in the deformation bands in front of the Lüders front. Figure 6 shows a set of images that exhibit the deformation band highlighted in Fig. 4(a). Because the specimen surface was mechanically polished after $5 \%$ deformation, no slipinduced surface relief was observed, as shown in the secondary electron image (Fig. 6(b)). In the deformation band, martensite formation was confirmed, as shown in Fig. 6(c). Correspondingly, the EBSD phase map (Fig. 7(b)) shows a significant reduction in austenite fraction in the deformation bands. That is, one of the significant plasticity mechanisms in the deformation bands was deformation-induced martensitic transformation to the $\mathrm{BCC}$ phase, and no transformation occurred in the exterior of the deformation bands, which also agrees with the results of a previous study on Lüders deformation in another medium-Mn steel. ${ }^{16)}$

Figure 8 shows the high-resolution EBSD results of the region in between the deformation bands. The phase map (Fig. 8(a)) shows an austenite fraction of $20 \%$. That is, no significant martensitic transformation occurred, which is consistent with the results shown in Fig. 7(b). Furthermore, the image quality value, which generally decreases with an introduction of dislocations ${ }^{24)}$ (although it is not solely dependent on $\operatorname{strain}^{25}$ ), was homogeneous distributed in the respective phases, indicating that dislocation slip also did not occur significantly in both ferrite and austenite regions between the deformation bands. This agrees with the results of a previous study on an $\mathrm{Fe}-10 \mathrm{Mn}-0.05 \mathrm{C}-1.6 \mathrm{Al}$ mediumMn steel. ${ }^{16)}$ In contrast, austenite fraction was reduced to 8\%, as shown in Fig. 9(a), where the deformation band

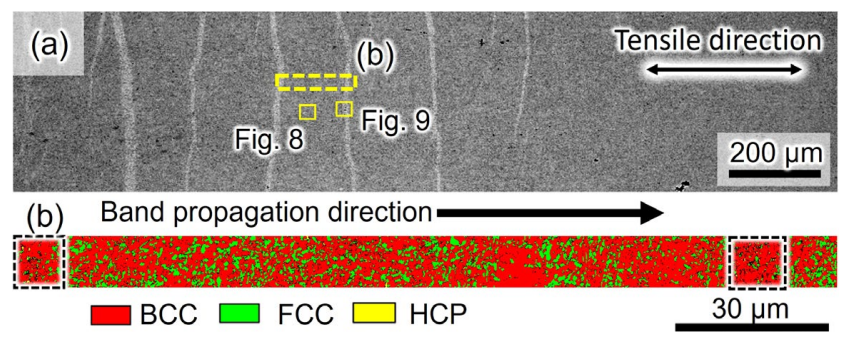

Fig. 7. (a) ECC image highlighted in Fig. 4(a). (b) Phase map of the region indicated in (a). The beam step size is $50 \mathrm{~nm}$. The regions where confidence index is below 0.1 are shown in black. (Online version in color.)
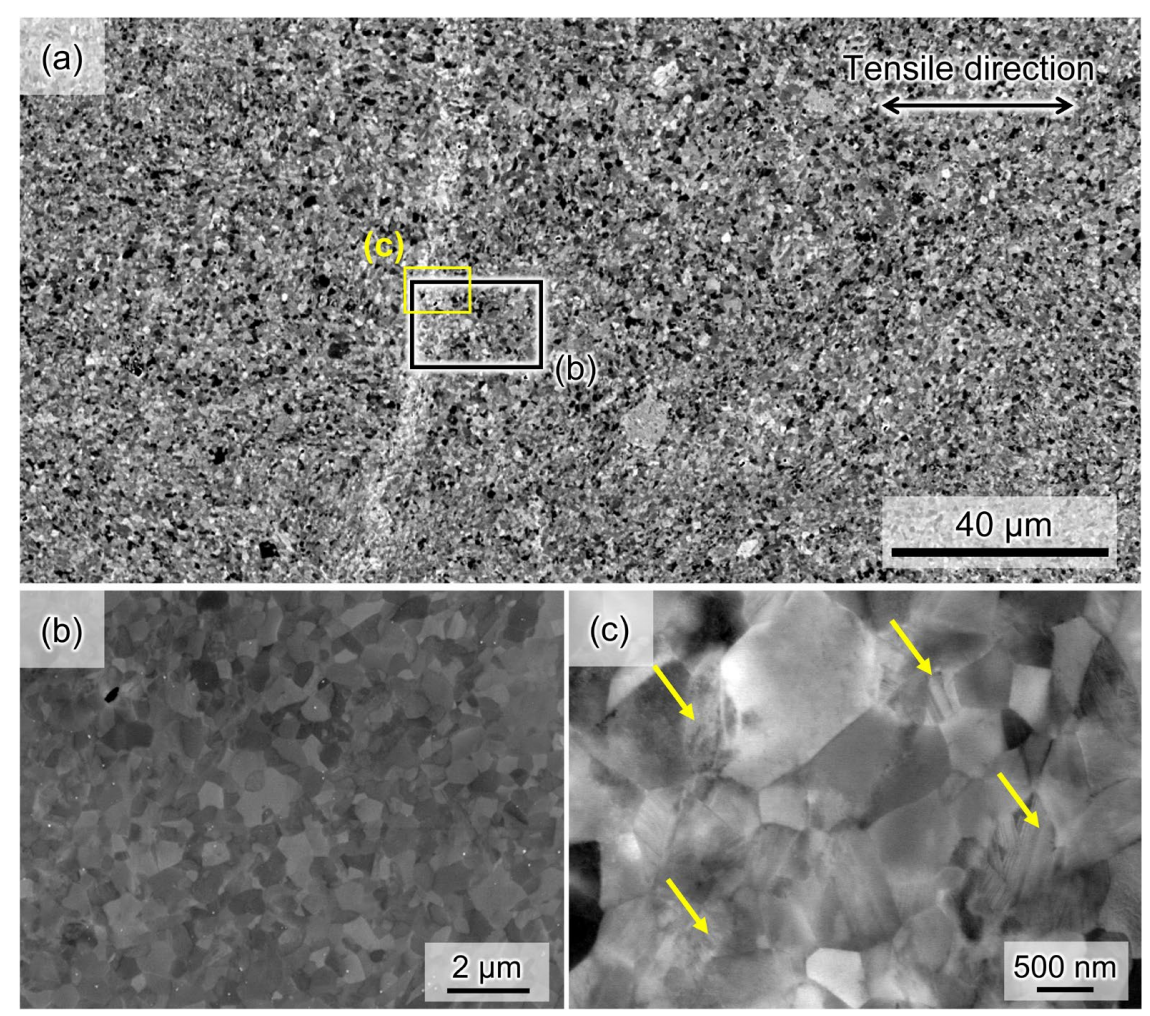

Fig. 6. (a) ECC images showing the coarse deformation band highlighted in Fig. 4(a). (b) Magnified secondary electron and (c) ECC images of the locations indicated in (a). The yellow arrows indicate martensite. (Online version in color.) 


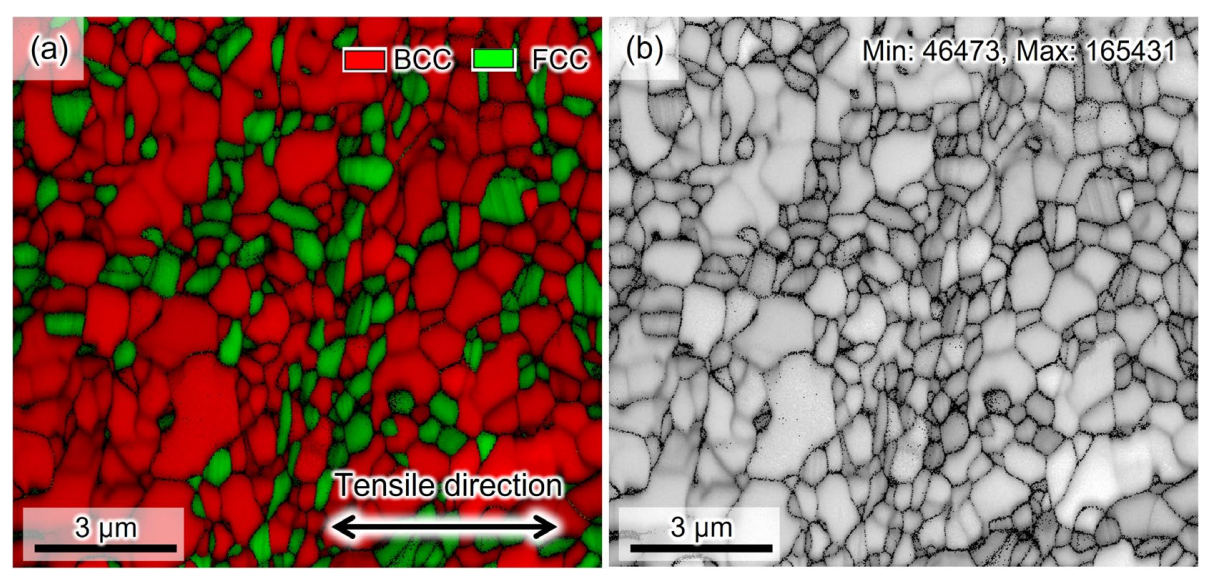

Fig. 8. (a) Phase and (b) image quality maps in the region between two deformation bands as highlighted in Fig. 7(a). The beam step size is $20 \mathrm{~nm}$. The regions where confidence index is below 0.1 are show in black. The displayed values in (b) indicate actual minimum and maximum image qualities. The values for scaling the gray contrast are set at 7857 and 166743 for minimum and maximum values, respectively, which are identical to the actual minimum and maximum values shown in Fig. 9(b). (Online version in color.)
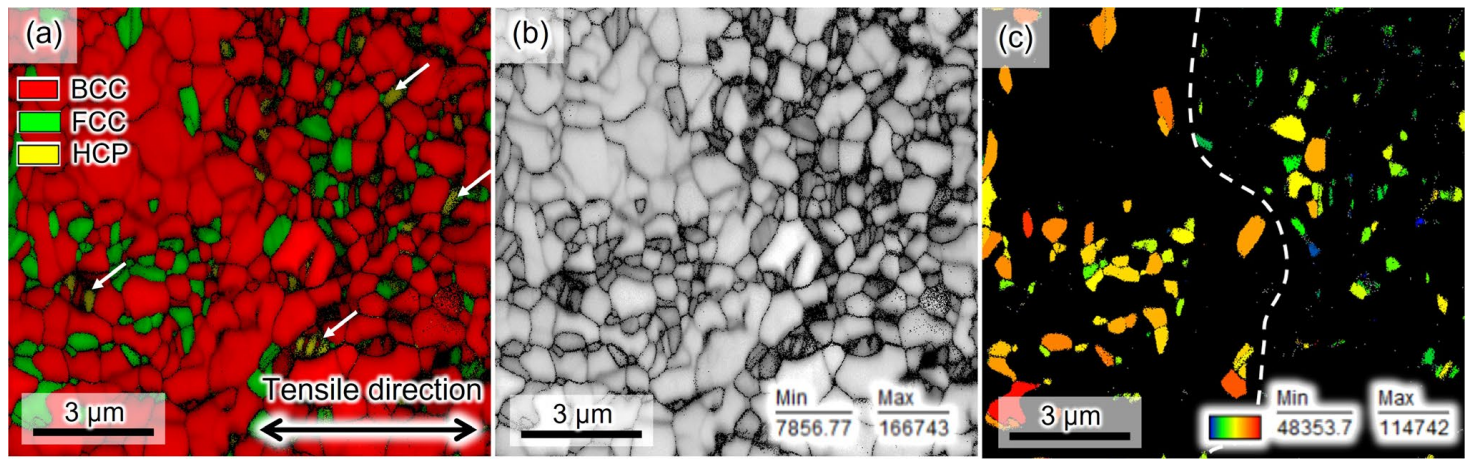

Fig. 9. (a) Phase and (b) image quality, and (c) grain average image quality maps in the region highlighted in Fig. 7(a). The right parts of the images contain the deformation band. The beam step size is $20 \mathrm{~nm}$. The regions where confidence index is below 0.1 are shown in black. (Online version in color.)

propagated in the right half of the image. In the corresponding image quality map (Fig. 9(b)), a remarkable reduction in image quality was observed. Since martensite contains a large number of dislocations, the lowest image quality was observed in the $\mathrm{BCC}$ martensite regions. Interestingly, the pre-existing $\mathrm{BCC}$ phase, which was mainly composed of ferrite, did not show any significant reduction in image quality even in the deformation band region, whereas the untransformed austenite did. For the sake of clarity, the grain-average image quality map of the untransformed austenite is shown in Fig. 9(c). It is clearly seen that the values in the right half region were lower than that in the left half region. That is, austenite was preferentially deformed even when deformation-induced martensitic transformation did not occur. This is also consistent with the results of a previous study that suggested that the dynamic strain aging in medium-Mn steels occurs via localized plasticity in untransformed austenite. ${ }^{4)}$

To prove that ferrite was a minor plasticity evolution site and that untransformed austenite acted as a preferential deformation site, an in-situ neutron diffraction experiment under tensile deformation was performed. Figure 10 shows the variation in the full width at half maximum (FWHM) of the diffraction peaks plotted against tensile strain, which qualitatively corresponds to the plasticity evolution. ${ }^{26)}$ It is obvious that the FWHM of the FCC phase (austenite) increased continuously, indicating that the dislocation density of the untransformed austenite increased with strain. On the other hand, the increment of FWHM in the BCC phase by straining was significantly lower than that in the FCC phase. Because the BCC phase contains martensite that includes a considerable number of dislocations, only a slight increase in FWHM indicates that the plasticity evolution in ferrite was not significant compared with that in the FCC phase. This result supports the fact that ferrite is a minor deformation site, as observed in Fig. 9(c).

Comparing the present results with those of previous studies, the role of ferrite in the plasticity during Lüders deformation in the cold-rolled and intercritically annealed medium-Mn steels depends significantly on the composition or microstructure. In the case of Fe-10Mn-0.05C-1.6Al steel annealed at $650^{\circ} \mathrm{C},{ }^{16}$ the behavior of the plasticity evolution in ferrite was similar to that in the present study, i.e., the ferrite was a minor plastic deformation site. In contrast, for the $\mathrm{Fe}-7 \mathrm{Mn}-0.1 \mathrm{C}$ steel annealed at $600^{\circ} \mathrm{C},{ }^{12)}$ although the microstructure was similar to that of the present steel, the yielding involving Lüders deformation was attributed to plasticity evolutions in both ferrite and austenite. The factors governing the local plasticity are considered as follows: (1) grain size, (2) austenite connectivity, (3) solution hardening degrees of the respective phases, (4) austenite phase stability effect on yielding, and (5) initial dislocation 
density. The austenite characteristics in the three steels are summarized in Table 1. Among these factors, the grain sizes of the Fe-10Mn-0.05C-1.6Al, Fe-7Mn-0.1C steels, and the present steels showed no significant differences. In addition, Wang et al. reported ${ }^{15)}$ that the local plasticity during Lüders deformation is insensitive to grain size and crystallographic orientation. The austenite connectivity in this study was lower than that of the other two steels because the austenite fraction was half that of the other two steels. Therefore, the preferential deformation in austenite must have occurred more easily in the Fe- $7 \mathrm{Mn}-0.1 \mathrm{C}$ steel than that in the present steel, in terms of austenite connectivity. Hence, the effects of grain size and austenite connectivity cannot be the reason that the significant deformation of ferrite in the Fe-7Mn-0.1C steel. Regarding solution hardening, the pres-
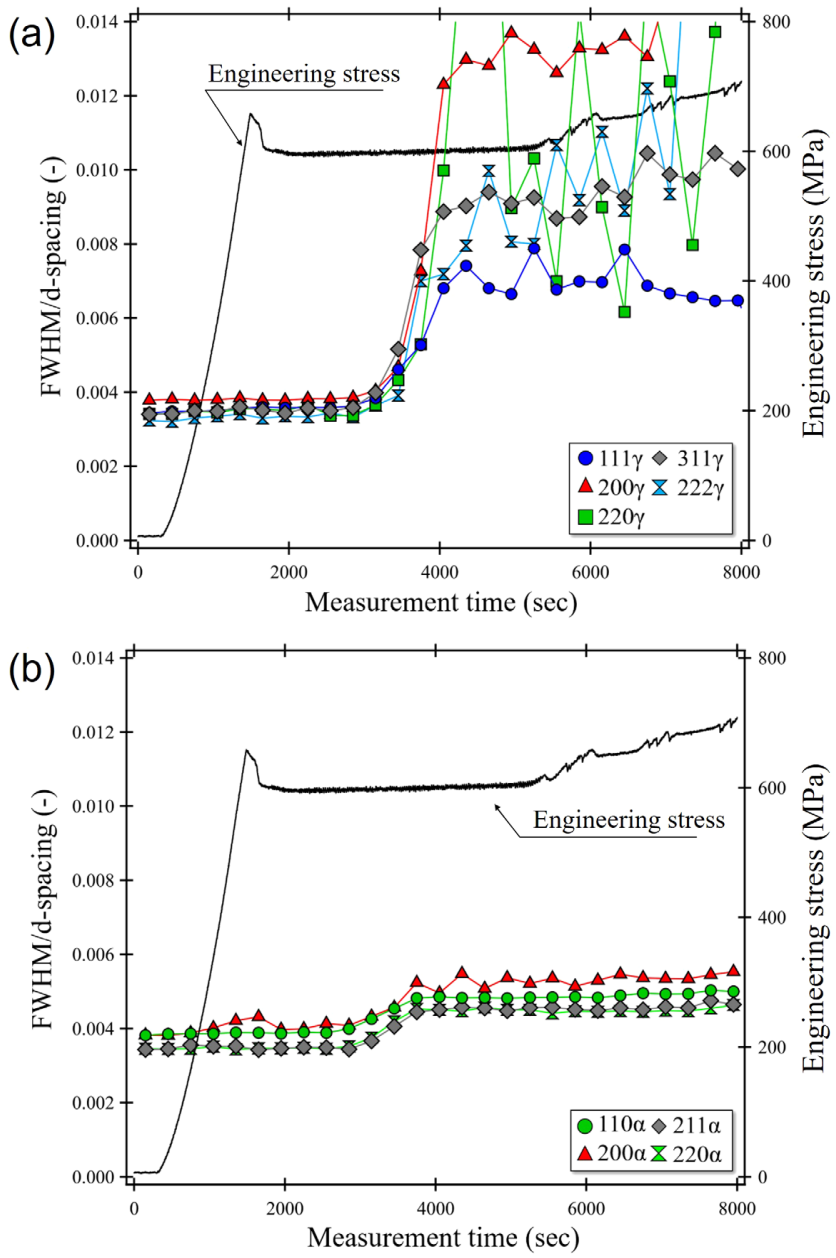

Fig. 10. Full width at half maximum plotted against measurement time in (a) FCC $(\gamma)$ and (b) BCC $(\alpha)$ phases. (Online version in color.) ences of $\mathrm{Al}$ and $\mathrm{C}$ harden ferrite and austenite, respectively. Therefore, the addition of $\mathrm{Al}$ and lower content of $\mathrm{C}$ in the Fe-10Mn-0.05C-1.6Al can show harder ferrite and softer austenite compared with those of the Fe-7Mn-0.1C steel, which is consistent with the fact that ferrite in the Fe-7Mn$0.1 \mathrm{C}$ was significantly deformed. However, because carbon content of austenite in the present steel must be higher than that of the Fe-7Mn-0.1C steel (the same nominal carbon content with the half austenite fraction), the present steel also must have shown ferrite deformation, which did not agree with the present results. From a viewpoint of phase stability, a low austenite stability decreases the critical stress for martensitic transformation, which decreases the yielding stress of austenite when it is controlled by martensitic transformation. In this context, we note the chemical compositions of the austenite in the Fe-10Mn-0.05C-1.6Al and $\mathrm{Fe}-7 \mathrm{Mn}-0.1 \mathrm{C}$ steels. The amount of $\mathrm{Mn}$ in austenite was estimated/measured to be 13 wt. $\%$ for both the steels. ${ }^{12,16)}$ Therefore, assuming that $\mathrm{Al}$ has only a minor effect, the double amount of $\mathrm{C}$ in the $\mathrm{Fe}-7 \mathrm{Mn}-0.1 \mathrm{C}$ steel must result in a higher austenite phase stability than that of the Fe-10Mn$0.05 \mathrm{C}-1.6 \mathrm{Al}$ steel. Hence, the effects of phase stability and the associated martensitic transformation on yielding can explain the preferential plasticity evolution in ferrite of the Fe-7Mn-0.1C steel. However, similar to the case of solution hardening, the effect of phase stability on yielding also cannot be a comprehensive explanation of the local plasticity in the medium Mn steels, because austenite of the present steel contains the higher carbon concentration than that of the Fe-7Mn-0.1C steel. The remaining factor, which is initial dislocation density, can be a comprehensive interpretation of the local plasticity. According to Wang's work, ${ }^{15)}$ the local plasticity of ferrite during the Lüders deformation in a medium Mn steel is dependent on initial dislocation density of each grain. Specifically, presence of a significant number of dislocations in ferrite facilitates local plasticity evolution in ferrite. In this context, the lower intercritical annealing temperature in the $\mathrm{Fe}-7 \mathrm{Mn}-0.1 \mathrm{C}$ steel $\left(600^{\circ} \mathrm{C}\right)$ than those in the other steels $\left(650^{\circ} \mathrm{C}\right)$ is considered to result in a relatively high initial dislocation density in the as-annealed condition, which assists the evolution of ferrite plasticity during the Lüders deformation.

In summary, the present results indicate that major factors affecting local plasticity evolution during the Lüders deformation of medium $\mathrm{Mn}$ steels are dislocation mobility in austenite relative to that in ferrite, mechanical austenite phase stability, and initial dislocation density of ferrite.

Table 1. Comparison of the characteristics of retained austenite in three cold-rolled and intercritically annealed medium Mn steels with sub-micrometer-sized austenite grains.

\begin{tabular}{ccccccc}
\hline & $\begin{array}{c}\text { Heat treatment } \\
\text { temperature }\end{array}$ & $\begin{array}{c}\text { Austenite } \\
\text { fraction }\end{array}$ & $\begin{array}{c}\text { Mn content } \\
\text { in austenite }\end{array}$ & $\begin{array}{c}\text { Austenite } \\
\text { morphology }\end{array}$ & $\begin{array}{c}\text { Strain } \\
\text { path }\end{array}$ & Ref. \\
\hline Fe-10Mn-0.05C-1.6A1 & $650^{\circ} \mathrm{C}$ & $40 \%$ & 13 wt.\% & Blocky & Austenite & $16)$ \\
Fe-7Mn-0.1C & $600^{\circ} \mathrm{C}$ & $39 \%$ & 13 wt.\% & Blocky & $\begin{array}{c}\text { Ferrite and } \\
\text { austenite }\end{array}$ & $12)$ \\
$\begin{array}{c}\text { Fe-5Mn-0.1C } \\
\text { (Present steel) }\end{array}$ & $650^{\circ} \mathrm{C}$ & $21 \%$ & - & Blocky & Austenite & \\
\hline
\end{tabular}




\section{Conclusion}

In this study, we focused on the microstructure and plasticity evolution during Lüders deformation in cold-rolled and intercritically annealed Fe-5Mn-0.1C steel (wt.\%), i.e., the representative model alloy of medium-Mn steels. The results obtained were as follows:

(1) Similar to other medium-Mn steels, the Fe-5Mn$0.1 \mathrm{C}$ steel showed sub-millimeter-scale deformation discontinuity, preferential plasticity evolution in retained austenite, and deformation-induced martensitic transformation during the Lüders deformation.

(2) The sequence of the deformation-induced martensitic transformation was $\mathrm{FCC} \rightarrow \mathrm{HCP} \rightarrow \mathrm{BCC} / \mathrm{BCT}$.

(3) Coarse deformation bands were formed in front of the Lüders front, and fine deformation bands were formed between the coarse deformation bands.

(4) Pre-existing BCC phase, which was mainly ferrite, was a minor path for microplastic deformation, which was also supported by the in-situ neutron diffraction experiment.

(5) During Lüders deformation, no significant plasticity evolution occurred in the regions where no deformation band was formed.

These are similar to the behavior of $\mathrm{Fe}-10 \mathrm{Mn}-0.05 \mathrm{C}$ 1.6Al steel, but dissimilar to that of the Fe-7Mn-0.1C steel, which is perhaps due to the difference in initial dislocation density.

\section{Acknowledgements}

This work was supported by JSPS KAKENHI (JP20H02457). The neutron diffraction experiments were performed at BL19 in the Materials and Life Science Experimental Facility of J-PARC (proposal No. 2019P0600).

\section{REFERENCES}

1) Y.-K. Lee and J. Han: Mater. Sci. Technol., 31 (2015), 843. https:// doi.org/10.1179/1743284714Y.0000000722

2) Y. Zhang and H. Ding: Mater. Sci. Eng. A, 733 (2018), 220. https:// doi.org/10.1016/j.msea.2018.07.052

3) H. Wang, Y. Zhang, G. Yuan, J. Kang, Y. Wang, R. D. K. Misra and G. Wang: Mater. Sci. Eng. A, 737 (2018), 176. https://doi. org/10.1016/j.msea.2018.09.045
4) J.-H. Nam, S.-K. Oh, M. Park and Y.-K. Lee: Acta Mater., 206 (2021), 116613. https://doi.org/10.1016/j.actamat.2020.116613

5) M. Callahan, O. Hubert, F. Hild, A. Perlade and J.-H. Schmitt: Mater. Sci. Eng. A, 704 (2017), 391. https://doi.org/10.1016/j. msea.2017.08.042

6) E. O. Hall: Proc. Phys. Soc. B, 64 (1951), 742. https://doi. org/10.1088/0370-1301/64/9/302

7) J. F. Butler: J. Mech. Phys. Solids, 10 (1962), 313. https://doi. org/10.1016/0022-5096(62)90003-0

8) S. Gao, M. Chen, S. Chen, N. Kamikawa, A. Shibata and N. Tsuji: Mater. Trans., 55 (2014), 73. https://doi.org/10.2320/matertrans. MA201317

9) N. Tsuji, Y. Ito, Y. Saito and Y. Minamino: Scr. Mater., 47 (2002), 893. https://doi.org/10.1016/S1359-6462(02)00282-8

10) M. Koyama, T. Sawaguchi and K. Tsuzaki: Mater. Sci. Eng. A, 530 (2011), 659. https://doi.org/10.1016/j.msea.2011.10.038

11) S. Gao, Y. Bai, R. Zheng, Y. Tian, W. Mao, A. Shibata and N. Tsuji: Scr. Mater., 159 (2019), 28. https://doi.org/10.1016/j. scriptamat.2018.09.007

12) B. C. De Cooman, P. Gibbs, S. Lee and D. K. Matlock: Metall. Mater. Trans. A, 44 (2013), 2563. https://doi.org/10.1007/s11661013-1638-6

13) M. Zhang, R. Li, J. Ding, H. Chen, J.-S. Park, J. Almer and Y.-D. Wang: Mater. Res. Lett., 6 (2018), 662. https://doi.org/10.1080/2166 3831.2018.1530698

14) X. G. Wang, L. Wang and M. X. Huang: Mater. Sci. Eng. A, 674 (2016), 59. https://doi.org/10.1016/j.msea.2016.07.054

15) X. G. Wang, C. H. Liu, B. B. He, C. Jiang and M. X. Huang: Mater. Sci. Eng. A, 761 (2019), 138050. https://doi.org/10.1016/j. msea.2019.138050

16) R. S. Varanasi, S. Zaefferer, B. Sun and D. Ponge: Mater. Sci. Eng. $A, 824$ (2021), 141816. https://doi.org/10.1016/j.msea.2021.141816

17) R. L. Miller: Metall. Mater. Trans. B, 3 (1972), 905. https://doi. org/10.1007/BF02647665

18) T. Furukawa, H. Huang and O. Matsumura: Mater. Sci. Technol., 10 (1994), 964. https://doi.org/10.1179/mst.1994.10.11.964

19) H. Luo, H. Dong and M. Huang: Mater. Des., 83 (2015), 42. https:// doi.org/10.1016/j.matdes.2015.05.085

20) Y. Ma: Mater. Sci. Technol., 33 (2017), 1713. https://doi.org/10.108 0/02670836.2017.1312208

21) J.-H. Nam, J. Han and Y.-K. Lee: Metall. Mater. Trans. A, 51 (2020), 1098. https://doi.org/10.1007/s11661-019-05570-w

22) S. Harjo, T. Ito, K. Aizawa, H. Arima, J. Abe, A. Moriai, T. Iwahashi and T. Kamiyama: Mater. Sci. Forum, 681 (2011), 443. https://doi. org/10.4028/www.scientific.net/MSF.681.443

23) R. Oishi, M. Yonemura, Y. Nishimaki, S. Torii, A. Hoshikawa, T. Ishigaki, T. Morishima, K. Mori and T. Kamiyama: Nucl. Instrum. Methods Phys. Res. Sect. A, 600 (2009), 94. https://doi.org/10.1016/j. nima.2008.11.056

24) S.-S. Rui, Q.-N. Han, X. Wang, S. Li, X. Ma, Y. Su, Z. Cai, D. Du and H.-J. Shi: Mater. Today Commun., 27 (2021), 102445. https:// doi.org/10.1016/j.mtcomm.2021.102445

25) S. I. Wright, M. M. Nowell and D. P. Field: Microsc. Microanal., 17 (2011), 316. https://doi.org/10.1017/S1431927611000055

26) S. Morooka, Y. Tomota and T. Kamiyama: ISIJ Int., 48 (2008), 525. https://doi.org/10.2355/isijinternational.48.525 